\title{
ON THE ORDER OF MAGNITUDE OF JACOBSTHAL'S FUNCTION
}

\author{
by R. C. VAUGHAN \\ (Received 5th January 1976)
}

Let $n$ be an integer with $n>1$. Jacobsthal (3) defines $g(n)$ to be the least integer so that amongst any $g(n)$ consecutive integers $a+1, a+$ $2, \ldots, a+g(n)$ there is at least one coprime with $n$. In other words, if

then

$$
G(j)=\min _{a} \sum_{\substack{m=a+1 \\(m, n)=1}}^{a+j} 1,
$$

$$
g(n)=\min _{G(j)>0} j
$$

It is probably true that

$$
g(n) \ll \omega(n)^{1+\epsilon}
$$

where $\omega(n)$ denotes the number of different prime divisors of $n$, and Erdós (1) has pointed out that by the small sieve it is possible to show that there is a constant $C$ such that

$$
g(n) \ll \omega(n)^{c} .
$$

The purpose of this short note is to show that in (4) $C$ can be taken arbitrarily close to 2 . Iwaniec (2, Theorem 2 ) has shown this in the special case when $n$ is the product of the first $r$ primes in order of magnitude. Any further substantial progress towards (3) probably requires a fundamental new idea. That 2 is the best that can be done by the method below is due to the usual inability of the one dimensional sieve to handle moduli larger than about the square root of the length of the interval under examination. Moreover, that any further improvement must lie very deep is indicated by the work of Kanold (4), (5) which shows that Linnik's celebrated theorem on the least prime in arithmetic progression follows easily from (4) with $C<2$.

Theorem. Suppose that $n>1$. Then

$$
g(n) \ll \omega(n)^{2}(\log 2 \omega(n))^{4} .
$$

Proof. Write

$$
r=\omega(n)
$$


and let

and

$$
h=\left[e^{2 A} r^{2}(\log 2 r)^{4}\right]
$$

$$
X=h^{\frac{1}{2}} e^{-A}
$$

where $A$ is a sufficiently large constant. Clearly it can be supposed that $r>r_{0}(A)$. The first step in the proof is to compare $n$ with

$$
P=P(X)=\prod_{p<X} p
$$

Let

$$
T=\sum_{\substack{m=a+1 \\(m, n)=1}}^{a+h} 1
$$

and

$$
S=\sum_{\substack{m=a+1 \\(m, P)=1}}^{a+h} 1 .
$$

Consider those $m$ counted in (10). For each such $m$, by $(8)$, either $(m, n)=1$ or there exists a prime number $p$ such that $p \mid(m, n)$ and $p>X$. Hence, by (9),

$$
S \leqslant T+\sum_{\substack{p>X \\ p \mid n}} \sum_{\substack{m=a+1 \\ p \mid m}}^{a+h} 1 \leqslant T+\sum_{\substack{p>X \\ p \mid n}}(h / p+1)
$$

Thus, by (5),

$$
T \geqslant S-r(h / X+1) .
$$

By (6), (7), (10) and the Corollary to Theorem 1 of Iwaniec (2) (the Rosser sieve),

$$
S \gg A h / \log ^{2} h
$$

since $A$ is sufficiently large. Hence, by (6), (7) and (11),

$$
\begin{aligned}
T & \geqslant S+O\left(h^{\frac{1}{2}} r e^{A}\right) \\
& \geqslant A h / \log ^{2} h
\end{aligned}
$$

again using that $A$ is sufficiently large. This completes the proof of the theorem.

\section{REFERENCES}

(1) P. ERDõs, On the integers relatively prime to $n$ and on a number-theoretic function considered by Jacobsthal, Math. Scand.10 (1962), 163-170.

(2) H. IWANIEC, On the error term in the linear sieve, Acta Arithmetic 19 (1971), 1-30. 
(3) E. Jacobsthal, Über Sequenzen ganzer Zahlen von denen keine zu n teilerfremd ist, I-III, Norske Videnske. Selsk, Forh. Trondheim 33 (1960), 117-139, IV, ibid. 34 (1961), 1-7, V, ibid. 34 (1961), 110-115.

(4) H.-J. KANOLD, Über Primzahlen in arithmetischen Folgen, Math. Ann. 156 (1964), 393-395, II, ibid. 157 (1965), 358-362.

(5) H.-J. KANOLD, Über eine zahlentheoretische Funktion von Jacobsthal, Math. Ann. 170 (1967), 314-326.

IMPERIAL COLLEGE,

LONDON, S.W.7. 